\title{
An Experimental Setup for Piezoelectric Cantilevered Vibration Energy Harvesters on a Rotating Drive Shaft
}

\author{
Dominik Gedeon, Stefan. J. Rupitsch \\ Chair of Sensor Technology \\ University Erlangen-Nuernberg (FAU), \\ Paul-Gordan-Str. 3/5, 91052 Erlangen, Germany \\ dominik.gedeon@fau.de
}

\begin{abstract}
:
We present an experimental setup along with a simulation environment for piezoelectric cantilevered Energy Harvesting structures under the influence of a stationary rotation. We explain the physical effects that lead to changes in the natural frequencies of the harvester. Consideration of the effects by the Finite Element Method is outlined. For the computation of damped eigenfrequencies, we exploit a model order reduction scheme based on a modal truncation technique, extended to piezoelectric structures. Parameters that influence the tuning behavior are identified and simulation based parameter studies are presented.
\end{abstract}

Key words: Piezoelectric Energy Harvesting, Vibration Energy Harvesting, Frequency Tuning, Finite Element Method, Modal Truncation.

\section{Introduction}

Mechanical vibration occurs as an unwanted byproduct in many technical processes. Rotating machinery is a prominent example for such a source of vibration. The physical causes for vibration in rotating assemblies are manifold. Imbalance or misalignment of shafts, Hooke's joints or reciprocating masses along with engine firing in combustion engines are common factors for torsional vibrations as well as bending and axial vibrations in drivetrains [1],[2]. Piezoelectric vibration Energy Harvesters (VEH) aim at converting this otherwise unused mechanical energy into electrical energy [3] [4]. As the conversion takes place at any location where vibration is present, electrical energy is readily available for sensors or other low-power electronics. Substitution of batteries or power transmission onto moving parts is a promising application field for vibration Energy Harvesters. When designing a linear oscillator-type mechanical structure incorporating piezoelectric material for energy conversion, the structure has to be optimized in accordance with the vibration characteristics of the source. Care must be taken to match a structure's resonance frequency to the harmonic component where mechanical vibration is most eminent. While vibration occurs at fixed frequencies in some applications, the aforementioned sources of vibration in rotating machines vary with revolution speed that, in many applications, is not constant [5].
Here, we consider a cantilevered piezoelectric energy harvester, which is mounted directly onto a rotating drive shaft. Due to the acceleration field, generated by the rotation, various mechanical forces other than the vibration itself, act on the structure, thereby causing a shift in resonance frequencies [6]-[9]. This tuning property must be accounted for in the design of a resonant electromechanical structure. In this contribution we present a simulation environment that is well suited to this task and that is capable of producing efficient numerical models for further investigations including electronic circuits, coupled to the piezoelectric structure [10]. We also present some validation results obtained by an experimental procedure for measuring the first bending mode eigenfrequency of a VEH under rotation.

\section{Physical Modeling}

Under the assumption of a rigid shaft, we model the harvesting structure in a non-inertial coordinate frame (Fig. 1). Furthermore, we consider the rotation to be stationary. Therefore, we can split the angular rate into a constant portion $\bar{\Omega}_{\text {rot }}$ and a time-varying part $\tilde{\Omega}_{\text {rot }}$, i.e.,

$\Omega_{\text {rot }}=\bar{\Omega}_{\text {rot }}+\tilde{\Omega}_{\text {rot }}$.

$\Omega_{\text {rot }}=2 \pi F_{\text {rot }}$

$F_{\text {rot }}$ is the rate of rotation in $\mathrm{Hz}$. In what follows, we assume the time-varying part to be small in comparison to the constant part. 
Figure 1 displays a cantilever with tip mass and a piezoelectric composite patch in a rotating environment. Rotation occurs around the z-axis of the non-inertial frame, causing a centrifugal acceleration field

$\mathbf{a}_{\mathrm{C}}=-\overline{\mathbf{\Omega}}_{\text {rot }} \times\left(\overline{\mathbf{\Omega}}_{\text {rot }} \times \mathbf{r}\right)$.

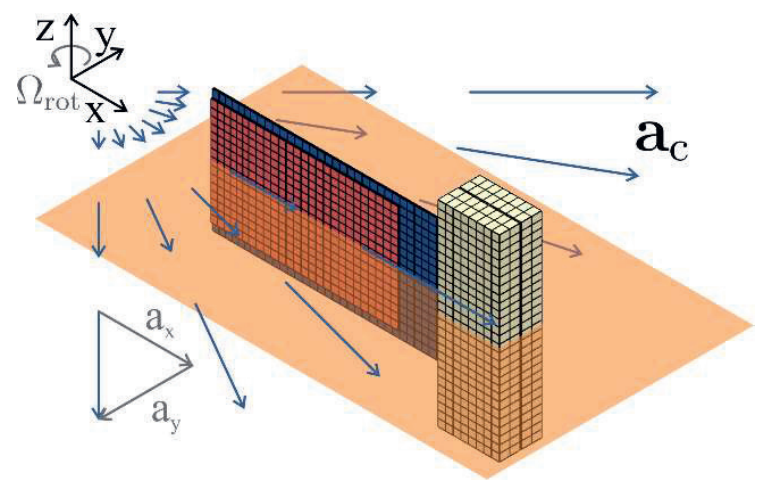

Fig. 1. FE model of a VEH with piezoelectric patch with centrifugal acceleration field. The free length of the harvester is $88 \mathrm{~mm}$, width $40 \mathrm{~mm}$, thickness 0.5 $\mathrm{mm}$. The patch is a PI DuraAct P-876.A12.

Here, $\overline{\mathbf{\Omega}}_{\text {rot }}$ is the vector of the rotation axis and $\mathbf{r}$ is the position vector. In Fig. 1 , the acceleration in longitudinal direction of the harvester, $a_{x}$, induces mechanical stresses in the material, thereby changing the stiffness of the structure. If the harvester performs a bending-mode vibration, in this example it will oscillate in $y$ direction. With $a_{y}$ being directly proportional to the $y$-coordinate of a given point, this effect acts as a negative stiffness on the structure, while displacements in z-direction are not affected. The orientation of a rotating structure, therefore, strongly influences its tuning behavior. If the vibration has a component perpendicular to the rotation axis, a Coriolis force $f_{\text {Cor }}$ will act on a moving mass $m$ that is proportional to its velocity v

$\mathbf{f}_{\mathrm{Cor}}=-2 m\left(\overline{\mathbf{\Omega}}_{\mathrm{rot}} \times \mathbf{v}\right)$.

Because of its velocity proportionality, the Coriolis effect can be modeled as direction dependent damping, potentially also affecting the tuning behavior.

In Fig. 2, a rotating cantilever sheet is depicted along with parameters of orientation that affect the tuning behavior. The angle $\Theta$ alters the direction of the bending mode vibration with respect to the rotation axis. Its value has an influence on the aforementioned negative stiffness as well as on the Coriolis force. The root offset $x_{\text {off }}$ from the rotation axis influences the magnitude of centrifugal forces acting on the structure in $\mathrm{x}$-direction.

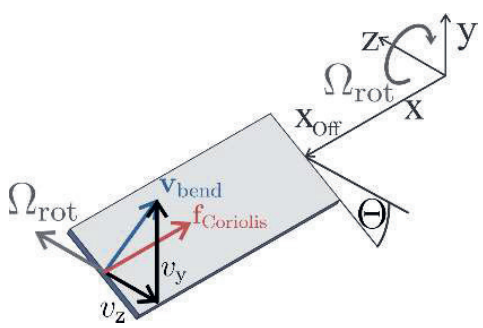

Fig. 2. Illustration of the parameters $\Theta$ and $x_{\text {off }}$ as well as the Coriolis force acting due to vibration and rotation.

\section{Numerical Modeling}

For numerical modeling of the piezoelectric structures under rotation, we used the Finite Element Method (FEM). All FE calculations were performed by the Finite Element code cfs++, which was developed at the Chair of Sensor Technology [11]. The change in stiffness due to the centrifugal acceleration is captured by the so-called geometrical stiffness that is generally dependent on the nodal displacements. Because of the stiffness being dependent on the displacements, the system has to be solved iteratively [12], i.e.,

$\mathbf{K}_{\mathrm{g}}\left({ }^{k} \mathbf{u}\right) \mathbf{s}=\mathbf{f}_{\mathrm{a}}$

${ }^{k+1} \mathbf{u}={ }^{k} \mathbf{u}+\mathbf{s}$

$\mathbf{K}_{\mathbf{g}}$ is the FE stiffness matrix, which depends on the nodal displacements $\mathbf{u}$ and the centrifugal acceleration forces $\mathbf{f}_{a}$ acting on the structure. The solution to (5) are the incremental displacements $\mathbf{s}$ at the $k^{\text {th }}$ iteration step. The nodal displacements are subsequently updated (6) and the procedure is repeated until a convergence criterion is met. As we consider the rotation to be stationary, (5) is static. In assuming the dynamic excitation of the harvesting structure to be small in comparison to the stationary centrifugal acceleration, a linearized dynamic computation around the solution of (5) is possible.

By direct coupling of the mechanical equations to the electrostatic field by the piezoelectric effect, we arrive at the piezoelectric dynamic system of ordinary differential equations:

$\left(\begin{array}{cc}\mathbf{0} & \mathbf{0} \\ \mathbf{0} & \mathbf{M}\end{array}\right)\left(\begin{array}{l}\ddot{\mathbf{V}} \\ \ddot{\mathbf{u}}\end{array}\right)+\left(\begin{array}{cc}\mathbf{0} & \mathbf{0} \\ \mathbf{0} & \mathbf{C}\end{array}\right)\left(\begin{array}{l}\dot{\mathbf{V}} \\ \dot{\mathbf{u}}\end{array}\right)+\left(\begin{array}{cc}-\mathbf{K}_{\mathrm{e}} & \mathbf{K}_{\mathrm{em}} \\ \mathbf{K}_{\mathrm{me}} & \mathbf{K}_{\mathrm{m}}\end{array}\right)\left(\begin{array}{l}\mathbf{V} \\ \mathbf{u}\end{array}\right)=$ $\left(\begin{array}{l}\mathbf{Q} \\ \mathbf{f}\end{array}\right)$

Here, $\mathbf{M}, \mathbf{C}$, and $\mathbf{K}_{\mathrm{m}}$ represent the mechanical mass, the damping and the stiffness matrices, respectively. The nodal force vector $f$ is the mechanical excitation. The nodal displacements are denoted by $\mathbf{u}$. A superscript dot indicates derivation with respect to time. $\mathbf{K}_{\mathrm{e}}$ is the electrostatic matrix and $\mathbf{V}$ are the electric nodal potentials. The electric right-hand-side consists 
of the nodal charges $\mathbf{Q}$. The electric and the mechanical parts of the system are coupled by the piezoelectric coupling matrices $\mathbf{K}_{\mathrm{em}}$ and $\mathbf{K}_{\mathrm{me}}$.

The direction-dependent negative stiffness due to the centrifugal acceleration is accounted for by adding an additional stiffness matrix $\mathbf{K}_{\mathbf{r}}$ to the mechanical stiffness matrix [13] resulting from (5)

$$
\mathbf{K}_{\mathbf{m}}=\mathbf{K}_{\mathrm{g}}+\mathbf{K}_{\mathrm{r}} \text {. }
$$

The velocity-dependent Coriolis effect is captured by the gyroscopic matrix $\mathbf{G}$, which is added to the Rayleigh model damping matrix $\mathbf{D}$

$$
\mathbf{C}=\mathbf{D}+\mathbf{G} \text {. }
$$

In order to efficiently solve the FE system (7), we exploit a modified modal truncation method for piezoelectric structures described in [10]. We solve the generalized eigenvalue problem for the coupled system

$$
\left(\begin{array}{cc}
-\mathbf{K}_{\mathrm{e}} & \mathbf{K}_{\mathrm{em}} \\
\mathbf{K}_{\mathrm{me}} & \mathbf{K}_{\mathrm{m}}
\end{array}\right) \boldsymbol{\phi}_{\mathrm{n}}=\boldsymbol{\omega}_{\mathrm{n}}^{2}\left(\begin{array}{cc}
\mathbf{0} & \mathbf{0} \\
\mathbf{0} & \mathbf{M}
\end{array}\right) \boldsymbol{\phi}_{\mathrm{n}}
$$

for a number of $N$ eigenvalues. This computation also yields the first $N$ eigenvectors

$$
\Phi=\left(\phi_{1}, \ldots, \phi_{N}\right)
$$

including the mechanical mode shapes as well as the corresponding electric potentials in the piezoelectric material. With the help of the mechanical and electrical parts of (11), all submatrices in (7) are truncated to a size of $N \times N$, exemplified here for the mechanical mass matrix M:

$$
\Phi_{\mathrm{m}}^{\mathrm{T}} \mathrm{M} \Phi_{\mathrm{m}}:=\mathrm{M}^{*} \text {. }
$$

The matrix of the first $\mathrm{N}$ mechanical mode shapes $\Phi_{m}$ is extracted from (11). A superscript ' $\mathrm{T}$ ' denotes the transpose of a matrix. By considering only a limited number of eigenvalues within the frequency range of interest, the order of the mathematical system is reduced significantly. In considering also the potential distributions for the electric parts of the system (7), the local properties of electric equations are preserved. The reduced system of equations can then be used to perform coupled simulations involving the harvesting structure as well as electronic circuits, e.g., in Simulink [10].

Here, we use the reduced system to solve a quadratic eigenvalue problem. The quadratic eigenvalue problem includes damping and, therefore, the Coriolis effect.

An automated simulation procedure was implemented that yields the eigenvalues, mode shapes and the reduced dynamic set of equations for a given number of angular rates
Rrot as well as for different combinations of geometrical and orientation parameters of the Energy Harvesting structure.

\section{Experimental Setup}

For an experimental assessment, we developed the setup, shown in Fig. 5a. It consists of a rotating shaft, driven by a brushless DC-motor with rated speed of $30001 / \mathrm{min}$. A piezoelectric VEH structure is mounted to the rotating structure, shown in more detail in Fig. 3. Electrical signals are transferred to and from the rotating shaft by means of a mercury-filled ring wheel (not shown). With the help of the variable mounting, the VEH is positioned with respect to the rotating axis, therefore varying the centrifugal and Coriolis forces, acting on the structure (see also Figs. 1 and 2). The first bending mode eigenfrequency is measured electrically under a constant rotational speed. Subsequently, the speed is increased and the next measurement is performed. In Fig. 4, the first bending mode eigenfrequency of the structure in Fig. 1 is plotted over rotational frequency for two different tip masses. In this experiment, the angle $\Theta$ was kept at $90^{\circ}$. The angle $\varphi$ remained at $0^{\circ}$.

\section{(a)}
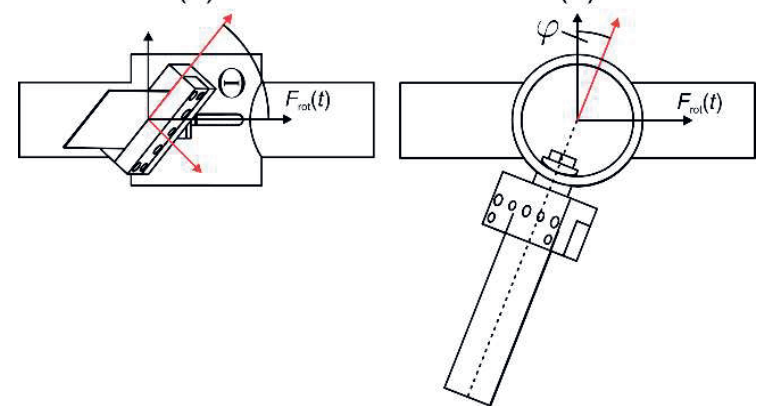

Fig. 3. Detail sketches of the mounting with VEH structure. (a): Side-view with angle $\Theta$. (b): Top-view with angle $\varphi$.

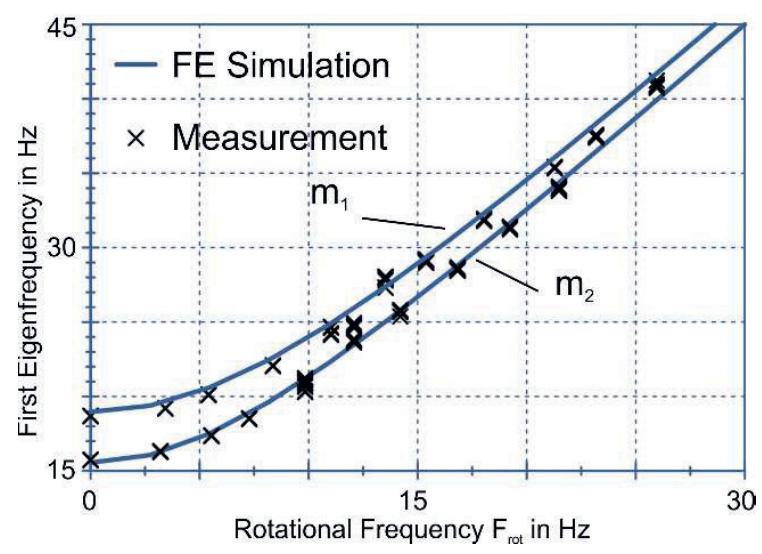

Fig. 4. First bending mode eigenfrequency of the cantilever VEH from Fig. 1 with piezoelectric patch and two different tip masses $m_{2}>m_{1}$. 
(a)

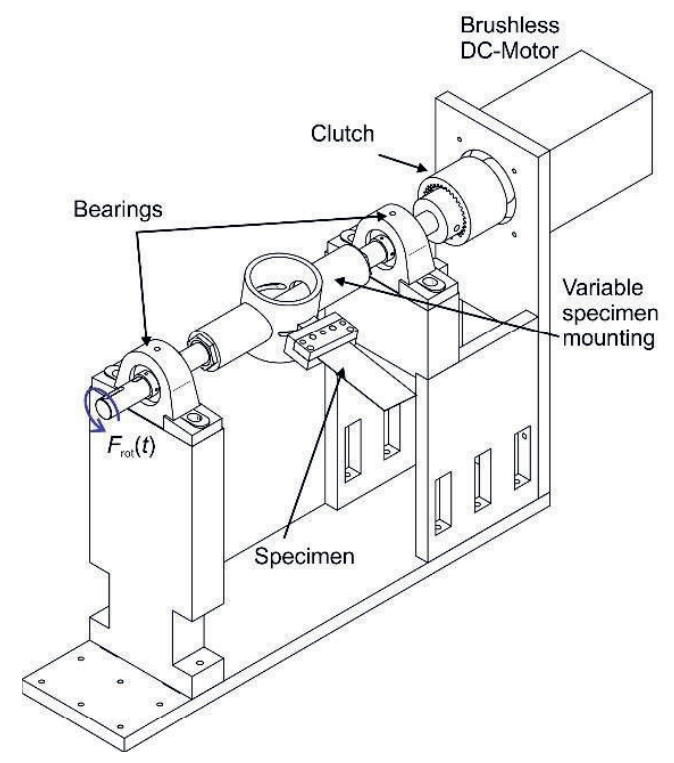

(b)

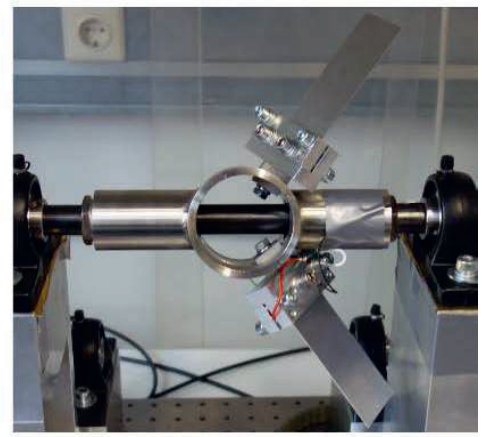

Fig.5. Experimental rotating setup. (a): Sketch of the mechanical part without housing around the mounting. (b): Photograph of the mounting with VEH structure and counterweight structure.

\section{Parameter Studies}

The eigenfrequencies of an energy harvesting structure under rotation as well as their tuning behavior are affected by various parameters. From Fig. 4 can be observed that an increase of the tip masses not only affect the natural frequency at $F_{\text {rot }}=0 \mathrm{~Hz}$, but additionally influences the steepness of the tuning curve.

The curves in Fig. $6 a$ are obtained from a simulation of a similar structure with two different tip masses. Additionally, the root-offset $x_{\text {off }}$ was varied. The sheet in Fig. 6a has a thickness of $0.5 \mathrm{~mm}$. In Fig. $6 \mathrm{~b}$, the thickness is increased to $1 \mathrm{~mm}$, while the other parameters remain unchanged.

In order to assess the influence of parameters on the steepness of the curves, we extracted the slopes at the highest rotational frequency of each curve by a finite difference. The result in Fig. 7 is the steepness $\frac{\Delta f}{\Delta F_{\text {rot }}}$ plotted over the height of the tip mass that is proportional to its weight. Additionally, the root-offset as well as the thickness were varied. It can be concluded from Fig. 7 that the steepness of the tuning curves is not monotonic with the weight of the tip mass. It is, however, remarkably affected by it. Figure 8 displays a similar result over the height of the tip mass and the thickness of the cantilever metal sheet at a fixed root-offset of $X_{\text {off }}=15 \mathrm{~cm}$. Similar conclusions can be drawn from investigations of other parameter variations, such as the free length of the harvesting structure or the position of the piezoelectric layer. (a)

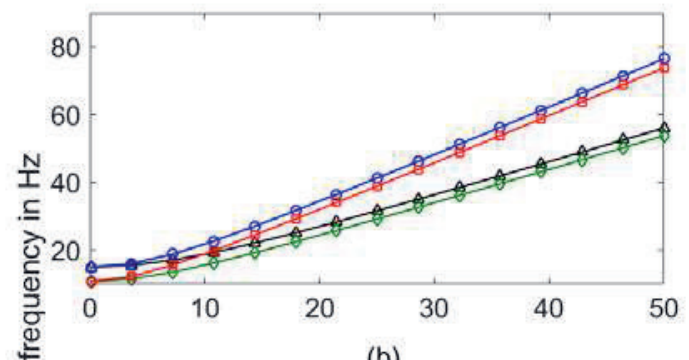

(b)

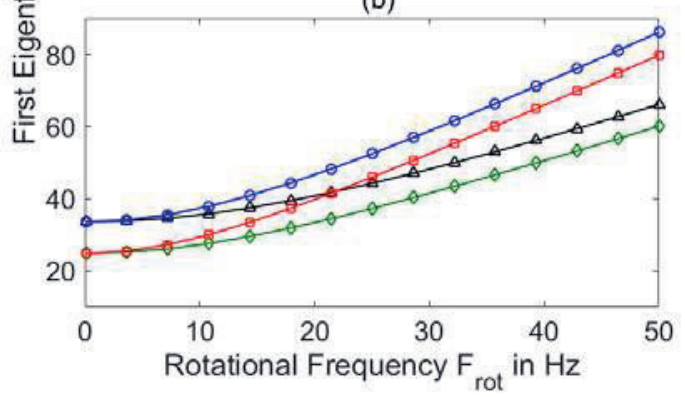

Fig. 6. First bending mode eigenfrequency of a cantilever VEH with piezoelectric patch and two different tip masses $m_{2}>m_{1}$ at two different root-offsets $x_{\text {off } 2}=5 \mathrm{~cm}, x_{\text {off1 }}=0$ (a): Thickness $0.5 \mathrm{~mm}$; (b): thickness $1 \mathrm{~mm} . \mathrm{X}: m_{1}, X_{\text {off2 }} ; \square: m_{2}, X_{\text {off } 2} ; \Delta: X_{\text {off } 1}, m_{1} ; \diamond: m_{2}$, Xoff1. 


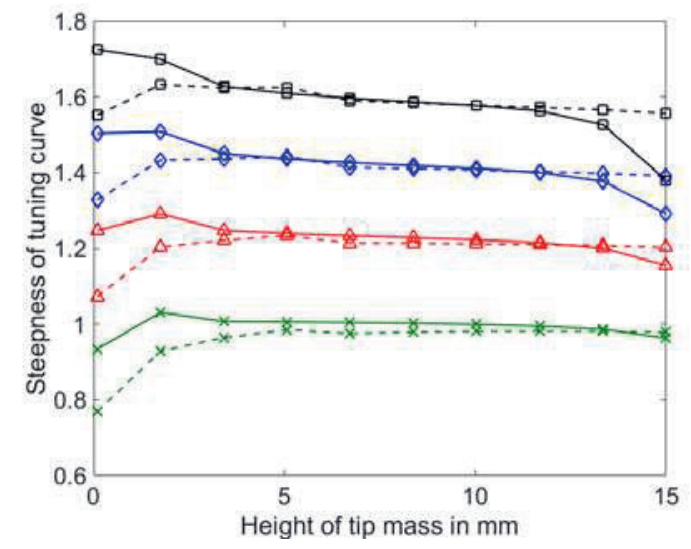

Fig. 7. Steepness of frequency tuning curves over height of the tip mass for different parameters. Solid lines: Thickness $1 \mathrm{~mm}$; dashed lines: thickness $0.5 \mathrm{~mm} ; \quad x: x_{\text {off } 1}=0 ; \Delta: x_{\text {off } 2}=5 \mathrm{~cm} ; \quad \Delta: x_{\text {off } 3}=10 \mathrm{~cm}$; $\square: x_{o f f}=15 \mathrm{~cm}$

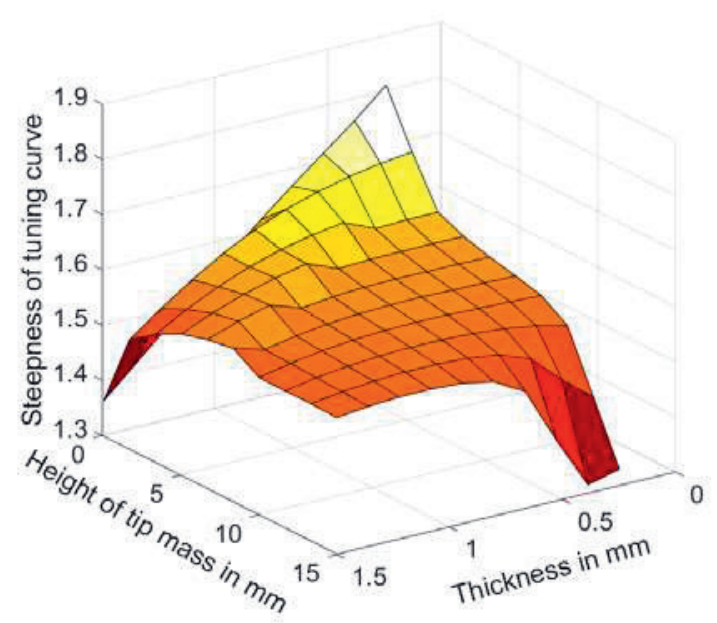

Fig. 8. Steepness of frequency tuning curves over height of the tip mass and thickness of the cantilever sheet with $x_{\text {off }}=15 \mathrm{~cm}$.

It has to be noted here that only one single structure, i.e., a straight cantilever with tip mass in clamped-free conditions with a piezoelectric patch, was investigated. The position of the patch as well as the shape of the harvester remained unchanged. It has been shown that the shape of a rotating structure also alters its tuning behavior [9].

\section{Conclusion}

We presented a FE based simulation environment for the numerical computation of piezoelectric energy harvesters undergoing a rotation. The simulation tool is part of a system simulation approach for energy harvesters that has been described previously. Furthermore, we introduced an experimental setup for the measurement of eigenfrequencies of energy harvesters under rotation along with some results, which coincide with the findings from simulations. Finally, we identified parameters that affect the tuning behavior in terms of steepness of the tuning curve. The influence of the angle $\Theta$ has yet to be validated by experimental measurements. Furthermore, the influence of the piezoelectric layer dimensions and position have to be taken into account in future studies.

\section{References}

[1] P. Couderc et. al., J. Sound Vib., vol. 218, no. 1, pp. 133-157, 1998

[2] Chikamori, S. et. al., JSAE Review, pp. 51-61, 1980

[3] H. A. Sodano et. al., J Intell Mater Sys Struct 16, 10 (2005), doi: 10.1177/1045389X05056681

[4] S. Roundy, J. Intel. Mat. Syst. Str., vol. 16, no. 10, pp. 809-823, 2005

[5] G. Rizzoni, IEEE Trans. Veh. Technol., vol. 16, no. 3, 1989

[6] A. Bokaian, J. Sound Vib., vol. 142, no. 3, pp. 481-458, 1990

[7] E. Chatelet et. al., J. Sound Vib., vol. 282, no. 12, pp. 163-178, 2005

[8] D. Gedeon et al., Ultrasonics Symposium (IUS), 2014 IEEE International, 2486-2489 (2014)

[9] H. Yoo et. al., J. Sound Vib., vol. 290, no. 1-2, pp. 223-241, 2006, doi: 10.1016/j.jsv.2005.03.014

[10] D. Gedeon et. al., ISAF, 2016 IEEE International, doi: 10.1109/ISAF.2016.7819733

[11] M. Kaltenbacher, Procedia Eng, vol. 5, 2010.

[12] K.-J. Bathe, Finite element procedures. KlausJurgen Bathe, 2006

[13] S.-H. Hsieh, J. Sound Vib, vol. 182, no. 1, pp. 91107, 1995 